Service social

\title{
Analyse des problèmes sociaux faite par la Commission Rochon. Sa portée et ses conséquences.
}

\section{André Beaudoin}

Volume 39, numéro 2, 1990

Les problèmes sociaux

URI : https://id.erudit.org/iderudit/706482ar

DOI : https://doi.org/10.7202/706482ar

Aller au sommaire du numéro

\section{Éditeur(s)}

École de service social de l'Université Laval

ISSN

1708-1734 (numérique)

Découvrir la revue

Citer cet article

Beaudoin, A. (1990). Analyse des problèmes sociaux faite par la Commission Rochon. Sa portée et ses conséquences. Service social, 39(2), 141-158.

https://doi.org/10.7202/706482ar

\section{Résumé de l'article}

Posant d'abord une distinction entre les problèmes sociétaux, qui sont d'ordre structurel, et les problèmes sociaux, qui sont associés à des personnes ayant des caractéristiques spécifiques ou à des groupes sociaux, l'auteur analyse la conception des problèmes sociaux qui se dégage du Rapport de la Commission d'enquête sur les services de santé et les services sociaux (Rapport Rochon) et les conséquences pour l'élaboration de l'action sociale et de la politique sociale. Ensuite, il montre comment la Commission, tout en prenant acte des multiples changements structurels qui ont caractérisé le Québec des dernières années (c'est-à-dire les problèmes sociétaux) s'oriente par la suite vers une analyse des problèmes sociaux. L'auteur situe la place de la pauvreté dans l'analyse développée par la Commission. Il explique pourquoi la Commission s'est orientée vers l'identification de groupes cibles : jeunes, familles monoparentales et personnes seules. Finalement, il résume l'analyse de la Commission pour ce qui est de l'identification des principaux problèmes de santé et des problèmes sociaux. 
André Beaudoin, professeur à l'École de service social de l'Université Laval et directeur du Laboratoire de recherche*.

\section{Analyse des problèmes sociaux faite par la Commission Rochon' Sa portée et ses conséquences}

André Beaudoin

Quelle conception des problèmes sociaux se dégage du Rapport de la Commission d'enquête sur les services de santé et les services sociaux (Rapport Rochon) ${ }^{2}$ ? Quelles sont les conséquences et la portée de l'analyse qui y est faite dans une perspective d'action sociale et de politique sociale ? Pour répondre à ces deux questions, nous examinerons d'abord les choix effectués par la Commission entre les dimensions sociales et sociétales dans son approche aux problèmes de santé et de services sociaux, ainsi que la nature des problèmes retenus par rapport à l'ensemble des problèmes existants. Notre analyse insistera sur l'extension et la gradation établies par la Commission dans les différents types de problèmes identifiés quant à leur signification et aux implications de ces choix pour les services sociaux.

\section{Des problèmes sociaux plutôt que des problèmes sociétaux}

Les fondements et les racines de ce que sont les problèmes de la société peuvent être présentés d'abord comme structurels, c'est-àdire à partir de la nature même de certaines caractéristiques fondamentales des structures et des modes de vie existant dans une société. Traditionnellement, une telle explication s'est fondée sur le marxisme. Plus récemment, se sont ajoutées l'explication féministe et celle de la sociologie des mouvements sociaux. La dimension structurelle est aussi présente à l'intérieur de la redéfinition du contrat social que sont en train de faire plusieurs sociétés ${ }^{3}$, redéfinition à laquelle n'échappe pas la société québécoise. On parle alors de 
problèmes sociétaux ou de problèmes macroscopiques ${ }^{4}$. Les problèmes sociétaux sont définis en lien avec les caractéristiques de la société dans son ensemble, les institutions établies et les grands mécanismes de fonctionnement qu'on y retrouve. On les analyse à partir des changements démographiques, des changements structuraux de l'économie, des changements dans la répartition de la richesse, des changements dans l'exercice des pouvoirs par certains groupes sur d'autres, etc. Cette conception des problèmes définis en interrelation avec des facteurs structurels a nécessairement des conséquences, dont la principale est de déboucher sur l'élaboration de solutions dans la perspective de la remise en cause de l'organisation même de la société.

Pourtant, une telle forme d'analyse est moins fréquente. On accepte assez difficilement de retracer la source des problèmes dans le fonctionnement même de la société dont la définition est faite par ceux-là mêmes qui occupent les positions de pouvoir et d'influence sur les structures. II n'existe pas non plus de façon bien définie d'exprimer quantitativement l'ampleur de certains problèmes sociétaux parce qu'il est difficile de les circonscrire, de les compiler, de les estimer et d'en faire l'exposé. Il est souvent même compliqué de les différencier des problèmes plus individuels ou microscopiques. Par exemple, on pourrait dire que les changements dans les structures familiales dans la société québécoise ont fait augmenter un certain nombre de problèmes comme ceux de la violence et des abus de toutes sortes, mais il est très difficile de vérifier un tel avancé, d'autant plus qu'il peut s'agir de la prise de conscience d'une réalité restée taboue auparavant.

$D^{\prime}$ 'une manière ou d'une autre, les problèmes qui sont l'objet de l'attention publique à un moment donné sont la résultante des valeurs de cette société. Ainsi, certaines situations prennent à la fois une plus grande visibilité tout en étant l'objet d'une augmentation réelle de leurs manifestations. En ce sens, il est difficile de distinguer entre des problèmes sociétaux, c'est-à-dire ceux qui sont d'ordre structurel dans une société, et des problèmes sociaux, c'est-à-dire ceux qui sont définis comme des caractéristiques plus particulières de personnes ou de membres de certains groupes. Tout au plus, est-il possible d'établir des liens entre les structures et les différentes situations problèmes lorsque ces dernières affectent une proportion assez importante d'une population pour se mériter l'attention publique et déboucher sur une action éventuelle.

Dans cette perspective, le point de départ de l'analyse faite par la Commission Rochon des problèmes existant au Québec est sociétal. L'analyse tient compte des changements structurels existant dans la société québécoise et les explicite. Elle décrit le Québec d'aujour- 
d'hui comme "une société en métamorphose $\|^{5}$ par rapport à toute une série de changements socio-économiques et politiques reliés à des facteurs comme les fluctuations de la croissance économique, l'augmentation de l'importance du rôle de l'État, les transformations du marché du travail et l'existence de nouvelles forces économiques, l'émergence de nouvelles formes de pauvreté, des changements sociodémographiques attribuables à la baisse de la natalité, au vieillissement de la population et à la composition multiethnique de la population, des changements de valeurs qui se reflètent dans la transformation de la famille, dans le mouvement des femmes vers l'égalité, dans l'émergence de nouvelles solidarités communautaires ou d'un nouveau dynamisme régional, et " $d^{\prime}$ une nouvelle manière de penser, de voir et d'agir en matière de santé ${ }^{6}$.

$C^{\prime}$ est donc dans le contexte de la société québécoise dans son ensemble que se situent les prémisses de départ de l'analyse des problèmes sociaux faite par la Commission, contexte d'une société qui est devenue pluraliste. Néanmoins, la Commission, ne fait pas à proprement parler l'analyse des problèmes vus comme sociétaux. Ceux-ci sont le plus souvent définis comme la résultante de choix individuels à l'intérieur de la société pluraliste du Québec. La Commission ne tente pas de situer l'ampleur des liens entre les transformations de la société québécoise et les problèmes existants, ou d'établir l'importance relative des transformations dans la détermination des problèmes. C'est pourquoi les problèmes constatés dans la société québécoise en matière de santé et de services sociaux sont d'abord analysés dans leurs manifestations concernant des individus, des groupes ou des catégories particulières de la population. Ils correspondent à la définition donnée voilà déjà quelques années par Etzioni à leur sujet : "Ce qui caractérise les problèmes sociaux c'est l'idée qu'ils sont considérés comme des conditions auxquelles il faut s'adresser pour les améliorer ou les maîtriser ${ }^{\prime}$. En ce sens, les problèmes abordés dans le rapport de la Commission sont des problèmes sociaux à l'égard desquels la société peut agir, plutôt que des problèmes sociétaux qui impliqueraient des changements structurels.

\section{Types de problèmes abordés}

La Commission avait un mandat relativement bien déterminé et délimité. On peut le résumer comme suit : évaluer le fonctionnement et le financement du système des services de santé et des services sociaux en regard d'objectifs plutôt circonscrits; étudier les différen- 
tes solutions possibles aux différents problèmes que connaît le système de santé et des services sociaux; faire les recommandations au gouvernement à leur égard ${ }^{8}$. Malgré un mandat qui faisait référence $d^{\prime}$ abord au fonctionnement du système, les commissaires ont néanmoins choisi de consacrer deux chapitres de leur rapport, l'un aux problèmes de santé et l'autre aux problèmes et aux besoins en matière de services sociaux. Ce choix est forcément limité et met de côté un ensemble d'autres problèmes sociaux avec lesquels est confrontée la société québécoise.

Parmi les problèmes que la Commission a mis de côté dans son analyse, les plus importants et les plus directement reliés à la santé et aux services sociaux sont certainement ceux de l'insuffisance des revenus et de l'insécurité économique et matérielle. À la différence de la Commission Castonguay-Nepveu qui avait considéré les problèmes reliés à la sécurité du revenu comme faisant partie des problèmes de base auxquels la société québécoise devait s'adresser au moment de la réforme en matière de santé et de bien-être ${ }^{9}$, la Commission Rochon ne touche pas directement à la dimension de la sécurité du revenu et des inégalités économiques existant dans la société québécoise. Malgré l'importance de ces problèmes, la Commission ne les inclut pas dans son analyse. Néanmoins, à plusieurs reprises, elle insère le problème de la pauvreté et des inégalités économiques comme faisant partie de l'intersection entre les problèmes de santé et de services sociaux. Ainsi, elle attribue aux conditions socio-économiques l'effet de la persistance des inégalités en matière de santé entre les groupes sociaux et dans les différents territoires et régions du Québec :

Elles [les inégalités sur le plan de la santé] correspondent aux inégalités sociales et démontrent de façon éloquente l'effet des divers déterminants environnementaux sur l'état de santé. Les groupes socialement défavorisés cumulent en effet un maximum de risques pour leur santé : ils sont davantage exposés à des conditions environnementales néfastes (conditions de travail, exposition aux polluants, habitation, niveau de revenus, etc.), ils ont des habitudes de vie dommageables (tabac, sédentarisme, mauvaise alimentation) et ont moins recours aux mesures préventives. Le cercle de la maladie et de la pauvreté se confirme encore ${ }^{10}$.

II en est de même dans le chapitre sur les problèmes en matière de services sociaux. La pauvreté y est considérée au même titre que les handicaps ou l'absence de réseaux d'intégration sociale, comme une condition de vulnérabilité qui affecte certains sous-groupes de la population et les rend plus susceptibles d'être aux prises avec certains problèmes en matière de services sociaux. La Commission cible certains groupes particuliers comme plus vulnérables sur le plan 
économique : les jeunes de moins de 25 ans, les familles monoparentales et les personnes seules. Elle établit un lien entre des conditions objectives de vie (par exemple le logement et l'alimentation) et des conditions de travail défavorisées comme étant la source de problèmes sociaux, à cause des circonstances éprouvantes qui les accompagnent. Pour la Commission, "la pauvreté implique non seulement la privation économique mais également la privation sociale, c'est-à-dire l'absence d'éducation, de considération, d'information. C'est donc dire que les pauvres disposent souvent de moins de ressources pour affronter les difficultés avec lesquelles ils sont confrontés. La pauvreté est donc à double titre un état de vulnérabilité ${ }^{11}$.

Malgré la reconnaissance de cette intersection, la Commission $s^{\prime}$ en est tenue dans son analyse à deux grands types de problèmes : les problèmes de santé et les problèmes de services sociaux. Nous donnerons dans les sections suivantes un bref aperçu des choix faits par la Commission en ce qui concerne les problèmes de santé, avant de traiter plus en détail par la suite des problèmes en matière de services sociaux.

\section{Définition et choix des problèmes en matière de santé}

L'approche choisie par la Commission dans la définition des problèmes de santé est à deux volets ${ }^{12}$. $\mathrm{D}^{\prime}$ un côté, elle continue de définir les problèmes de santé comme des pathologies; de l'autre, elle les voit dans une perspective où "l'état de santé d'une population est le résultat de l'interaction dynamique de quatre grandes catégories de facteurs : les facteurs biologiques, les habitudes de vie, les conditions environnementales et l'organisation du système de soins lui-même ${ }^{13}$.

En ce qui concerne les pathologies, la Commission attire l'attention sur les principales maladies qu'on retrouve au Québec et leur répartition : maladies cardiovasculaires, cancer, traumatismes, suicide, maladie du système locomoteur, maladies transmissibles sexuellement et sida, ainsi que des problèmes de santé mentale. Pour ce faire, elle dispose de données considérables qui fournissent une bonne vue d'ensemble de la répartition des problèmes de santé dans leurs perspectives pathologiques individuelles. Ainsi, la Commission fait très bien ressortir les pathologies physiques de même que leur incidence et leur prévalence au Québec. Par ailleurs, dans des problèmes comme ceux du suicide et de la santé mentale où bien souvent les constatations pathologiques doivent être reliées à 
d'autres facteurs externes, il est beaucoup plus difficile de situer l'analyse de la Commission. Elle demeure plus-imprécise et moins fondée sur des informations explicites.

La Commission dépasse toutefois I'explication des seules pathologies comme moyen d'interpréter l'état de santé pour faire ressortir d'autres facteurs reconnus comme de plus en plus importants dans l'explication des problèmes de santé. Le premier de ces facteurs, de nature biologique, fait intervenir plus particulièrement le domaine de la génétique et de la biologie cellulaire et moléculaire : il est à la base même des explications plus directes de plusieurs maladies. Actuellement, les recherches en cours peuvent contribuer à l'explication et à la solution de plusieurs problèmes de santé jusque-là restés sans réponse.

En deuxième lieu, les problèmes de santé sont aussi reliés aux comportements et aux habitudes de vie. L'identification de certains comportements et de certaines habitudes de vie comme étant des risques pour la santé implique une définition des problèmes de santé non plus seulement dans leurs dimensions pathologiques et biologiques, mais aussi dans leurs dimensions sociales. Il y a une liaison entre les actes et les conduites des individus eux-mêmes et leur état de santé. Les problèmes de santé deviennent des problèmes à caractère social parce qu'ils sont conçus dans leur rattachement à certains comportements des personnes dans la société. C'est ainsi que la Commission dépeint le tabagisme, l'alcoolisme et les autres toxicomanies comme reliés aux problèmes de consommation de certaines substances dommageables. Mais en même temps, les problèmes de santé peuvent être aussi liés à des habitudes de vie déficientes telles que l'absence d'activités physiques ou un régime alimentaire inadéquat.

En troisième lieu, les commissaires élargissent leur conception des problèmes de santé aux dimensions environnementales. C'est ainsi qu'ils identifient l'air, l'eau, le sol et la qualité des aliments, et même le milieu de travail, comme pouvant constituer des risques pour la santé. Mais on les sent en terrain beaucoup moins sûr à ce niveau. Ils parlent de la difficulté de cerner avec précision la définition même du terme environnement et des changements qui sont en train de se produire dans la nature des interventions gouvernementales. Ils soulignent la « marge d'incertitude et d'erreur lorsqu'il s'agit d'estimer l'ampleur et l'importance sur la santé des différents types de risques reliés à l'environnement $»^{14}$. En ce sens, la Commission reflète la difficulté dans laquelle on se retrouve de transposer sur le plan structurel, macroscopique et sociétal, l'analyse de certains problèmes de santé définis de façon individuelle, microscopique et pathologique. En effet, analyser les problèmes de santé à partir de leurs 
liens environnementaux, c'est faire intervenir les croyances, les valeurs et même le mode d'organisation de la société dans sa façon $d^{\prime}$ utiliser et d'explorer les ressources existantes en lien avec la santét ${ }^{15}$.

\section{La gradation des problèmes auxquels s'adressent les services sociaux}

Tout en constatant l'insuffisance des connaissances et des données en sa possession et la faiblesse de la recherche appliquée en la matière, la Commission a abordé la question des problèmes et les besoins en matière de services sociaux selon une gradation à trois paliers : les problèmes sociaux fondamentaux qui touchent à des groupes à risque, les conditions de vulnérabilité de certaines populations particulières et les difficultés courantes de fonctionnement de l'ensemble de la population ${ }^{16}$.

Dans cette perspective, on retrouve en filigrane de l'analyse faite par la Commission, une conception des problèmes où sont mises en cause des conditions à changer touchant la socialité, c'est-à-dire dans les rapports sociaux qui concernent les personnes dans leur conduite, dans leurs interactions et leurs relations sociales à l'intérieur des arrangements de la société définis comme sources d'échanges ${ }^{17}$.

\section{Les problèmes sociaux fondamentaux}

À ce premier palier, les problèmes auxquels les services sociaux s'adressent sont définis par la Commission comme fondamentaux à partir de trois critères d'observation : 1) leurs manifestations " atteignent un niveau grave et socialement intolérable $» ; 2$ ) ils produisent " des effets éventuels à court, moyen et long termes sur l'individu et la société " et "tendent à se répéter d'une génération à l'autre "; 3 ) « ils touchent largement les individus et les groupes qui ne peuvent faire face à leurs difficultés par leurs seuls moyens et ressources ${ }^{18}$. Ces critères permettent, jusqu'à un certain point, un parallèle que la Commission ne fait qu'implicitement avec la santé. Ce qui est défini comme un problème social "fondamental ", faute d'un meilleur terme dans les services sociaux, est en quelque sorte assez près de ce qu'on appelle un problème " pathologique ». II se rapporte au mal fonctionnement social des individus. Trois catégories de problèmes fondamentaux sont identifiées :

— Les problèmes de violence contre les personnes. Ils se définissent comme l'exercice abusif du pouvoir par une ou des personnes à l'égard d'autres personnes. Ils incluent en particulier la négli- 
gence, les abus et les mauvais traitements faits aux enfants, les agressions à caractère sexuel à l'égard des femmes, la violence conjugale et la violence faite aux personnes âgées. C'est l'intégrité physique et sociale de la personne elle-même qui est affectée dans ses rapports avec d'autres personnes dans la mesure où elle se trouve dans une situation sans défense et sujette à une manipulation abusive.

- La déviance et la mésadaptation sociale. La Commission fait une distinction entre déviance et mésadaptation sociale. Elle définit la première comme des comportements sociaux jugés inacceptables et qui «peuvent nécessiter l'intervention de l'État surtout s'ils mettent en jeu la sécurité d'un tiers et de la société $»^{19}$. C'est la délinquance des jeunes qui est l'objet principal de préoccupation de la Commission. Quant à la mésadaptation, elle est définie comme «certaines perturbations sérieuses du comportement ou certains modes réactionnels [qui] peuvent compromettre la sécurité, l'intégrité ou le développement de la personne ${ }^{20}$ : on y inclut les troubles sérieux de comportement qui échappent au contrôle des parents, l'itinérance, les tentatives de suicide et les tendances suicidaires à l'adolescence.

— Les problèmes reliés à l'intégration sociale ${ }^{21}$. L'intégration sociale est définie comme la participation autonome des individus et des groupes à la vie en société grâce à l'incorporation de modèles, de comportements, de normes et de valeurs. L'intégration se fait à travers l'entourage immédiat de la personne et le lien qui s'établit avec un univers social élargi. Les réseaux sociaux naturels ainsi constitués se trouvent composés d'un certain nombre de systèmes comme la famille, l'école, le travail, la parenté ou le voisinage. Il y a pour la Commission un problème social fondamental lorsque deux critères nécessaires à l'intégration sociale ne sont pas satisfaits : l'absence ou la carence de soutien de la part des réseaux naturels; la gravité, la persistance ou le cumul de certaines difficultés liées à cette absence. Quatre problèmes principaux d'intégration sociale sont retenus par la Commission : les carences du lien parental en ce qui concerne le développement de l'enfant, la surcharge de certaines familles monoparentales en matière d'obligations familiales, les difficultés liées à l'absence, à la perte ou à la diminution de l'autonomie fonctionnelle, et les problèmes d'intégration à la société que vivent les immigrants.

Dans son ensemble, la référence que fait la Commission à un premier degré de problèmes définis comme "fondamentaux" permet de positionner à l'avant-scène plusieurs problèmes sociaux qui découlent de nouvelles situations devenues plus manifestes dans 
la société québécoise au cours des dernières années. Si plusieurs de ces problèmes occupent souvent la première place dans les médias, il s'agit de problèmes qui demeurent, malgré tout, mal connus. La Commission n'a pu que référer à des données très souvent indirectes pour appuyer son analyse. Plusieurs pistes de recherche doivent être poursuivies pour mieux cerner les multiples dimensions de ces problèmes : l'influence de la famille et des réseaux primaires comme force stabilisatrice des comportements; les difficultés pour les jeunes couples de faire face aux obligations parentales en même temps qu'aux autres obligations reliées au travail, à l'école et à la vie en société; les effets sociaux de l'alcoolisme et des toxicomanies diverses; les problèmes de la réinsertion sociale des " ex-institutionnalisés ", etc. II s'agit là d'une gamme de problèmes qui, tout en faisant partie du cadre des problèmes fondamentaux de la Commission, nécessitent des recherches considérables pour être mieux connus et surtout faire l'objet d'une attention plus adéquate.

Dans l'identification faite des trois catégories de problèmes fondamentaux, on retrouve néanmoins des différences de niveau. Les problèmes de violence impliquent des personnes qui sont l'objet d'une attaque et dont la sécurité est mise en cause par une ou plusieurs personnes. II en découle que ce sont les victimes qui doivent être d'abord l'objet de l'attention des services sociaux; quant aux agresseurs, on est beaucoup moins explicite à leur sujet. Du fait qu'ils soient à la source des problèmes, comment doit-on les considérer ? Le danger demeure de se limiter à une attitude punitive à l'égard des agresseurs et à une attitude de protection à l'égard des victimes. Existe-t-il des liens entre les mécanismes de fonctionnement de l'entourage et de la société dans son ensemble et les agressions sexuelles ? Qu'est-ce qui est à l'origine des mauvais traitements et de la négligence à l'égard des enfants ? Comment prévenir et résoudre les situations de violence conjugale ? Où commencent les agressions contre les personnes âgées ? Ce sont là des questions qui doivent être l'objet d'études plus poussées.

Pour ce qui est des problèmes de déviance, la Commission n'a retenu que les problèmes de la délinquance des jeunes et elle a mis de côté la question de la délinquance et de la criminalité adultes. Les dimensions sociales de ce dernier problème sont pourtant considérables, mais la Commission ne possédait pas les données requises pour en faire l'analyse. Par ailleurs, pour définir les troubles de comportement en liaison avec la mésadaptation sociale, les critères utilisés sont ceux de la Loi sur la protection de la jeunesse. II est essentiel de connaître le phénomène au-delà des seules catégories légales. Pourquoi les troubles de comportement ne se manifestent-ils qu'après l'âge de douze ans et surtout après l'âge de quatorze ans ? 
Pourquoi ces jeunes proviennent-ils surtout de milieux défavorisés socio-économiquement? Comment se fait-il que plusieurs d'entre eux ont été antérieurement l'objet d'intervention des services sociaux ? Qu'est-ce qui est à l'origine d'un problème de trouble de comportement? Des questions du même genre se posent pour d'autres problèmes comme l'itinérance et les tentatives de suicide chez les jeunes.

En somme, les deux premières catégories de problèmes, ceux de la violence contre les personnes et ceux de la déviance et de la mésadaptation sociale, font référence à des phénomènes plutôt pathologiques sur le plan social, auxquels toute l'attention nécessaire doit être accordée parce qu'ils constituent une menace pour autrui et l'ensemble de la société. Ils exigent prévention, dépistage, intervention et solution un peu à la manière des problèmes pathologiques en santé. Malgré tout, la conception qu'on s'en fait peut facilement devenir très limitée et se ramener à la notion de risque et de groupe à risque, comme l'a fait jusqu'à un certain point le rapport Brunet ${ }^{22}$ pour situer l'orientation des interventions dans les CLSC ${ }^{23}$.

Dans la troisième catégorie de problèmes fondamentaux, c'est-àdire ceux reliés à l'intégration sociale, le schème de référence dépasse celui de la pathologie pour considérer les besoins liés aux conditions de vie en société. Ainsi, lorsqu'on fait référence aux carences de liens parents-enfants, on ne peut se contenter d'attendre pour faire face aux conséquences négatives des difficultés rencontrées en ce qui concerne les liens entre parents et enfants. Les familles où le père et la mère travaillent, les familles à parent unique et celles qui ne bénéficient pas de soutien dans leur entourage immédiat ont besoin de support et d'appui, de la part de la société pour pouvoir s'occuper de leurs enfants, les éduquer et les socialiser adéquatement. Il y a des enfants qui ont besoin de soins et d'attention pendant que les parents sont au travail. Plusieurs familles doivent avoir à leur disposition plus de moyens financiers pour pouvoir faire face à leurs obligations et aspirations, pour vivre avec un minimum de confort et fournir le support nécessaire au développement affectif et social de leurs enfants, en vue de leur développement comme citoyens participant à la société, peu importe leur milieu d'origine.

$C^{\prime}$ est de la même manière qu'il faut aussi considérer les difficultés des personnes atteintes d'une incapacité physique ou mentale à un degré ou l'autre. Déjà, au cours des dernières années, des efforts ont été faits pour fournir des réponses plus adaptées aux besoins précis de ces personnes avec la Loi sur les personnes handicapées. Si antérieurement on avait tendance à institutionnaliser plusieurs de ces personnes, le mouvement récent de désinstitutionnalisation et de vie dans le milieu des personnes souffrant d'une 
incapacité physique ou mentale met en relief la nécessité de continuer le développement des nouveaux mécanismes d'aide et d'appui, et des nouveaux modes d'organisation pour permettre aux personnes handicapées de participer selon toute leur capacité à la vie en société.

Le problème de l'intégration des immigrants à la société québécoise est aussi retenu par la Commission. Dans le contexte mondial des bouleversements démographiques qu'est en train de connaître la planète, le Québec, dont le taux de natalité est devenu l'un des plus bas au monde, est de plus en plus une terre d'accueil pour les immigrants et pour les réfugiés. Encore une fois, c'est à une nouvelle série de besoins auxquels la société québécoise doit s'adresser pour aider les nouveaux arrivants à s'intégrer de manière dynamique, afin de leur permettre de construire ou de reconstruire leurs réseaux sociaux et de s'adapter à un nouveau contexte dans plusieurs secteurs de leur vie (travail, consommation, habitudes, loisirs, etc.). Mais encore là, force est de constater que la Commission ne dispose pas de beaucoup de données sur l'intégration des immigrants à la société québécoise. Toute une série de questions à cet égard demeurent sans réponse. Pourquoi beaucoup d'immigrants utilisent-ils le Québec comme terre de passage plutôt que terre d'adoption ? Qu'est-ce qui fait que c'est dans le milieu urbain, à Montréal principalement, que demeurent les nouveaux arrivants ? À quoi les difficultés d'intégration sont-elles attribuables? Tout en faisant consensus sur le caractère fondamental de cette question, il reste beaucoup à faire pour mieux connaître la situation et surtout pour s'y adresser de façon proactive.

À partir du moment où les problèmes sont définis dans une perspective d'intégration sociale, on est forcé d'abandonner une approche où voisinent très souvent des attitudes de blâme à l'égard des victimes et une définition de leur situation à la lumière de critères de déficience et d'anormalité. On n'est plus uniquement en présence $d^{\prime}$ 'individus ou de groupes à risques dans une perspective de prévention et traitement de problèmes, mais certains aspects de la configuration même de l'organisation de la société sont aussi en cause. Ils doivent être changés dans la perspective de répondre aux besoins existants.

\section{La vulnérabilité de certaines populations}

Mais la Commission met de côté la seule considération des problèmes sociaux vus sous le prisme de leur gravité, de leur urgence et du caractère intolérable de certaines situations, pour déboucher d'une part sur les conditions de vulnérabilité que représentent des 
situations particulières de vie de certaines parties de la population et, d'autre part, sur les difficultés courantes de fonctionnement en société. Examinons d'abord la vulnérabilité. Elle est définie comme "l'ensemble des conditions qui font qu'un sous-ensemble d'individus dans une population particulière sont susceptibles de présenter des problèmes sérieux dans certaines situations adverses, devant le danger ou la malchance $»^{24}$. En attirant l'attention sur les conditions de vulnérabilité, on met en évidence les facteurs mêmes qui sont des préconditions à l'émergence des problèmes sociaux dans la sociétée 25 .

Comme société démocratique, de plus en plus pluraliste et à orientation humanitaire, la société québécoise est en train d'apprendre que certaines conditions sociales fournissent de meilleures garanties de stabilité et de bien-être pour l'ensemble de la population. D'autres conditions, au contraire, empêchent certains groupes de réaliser pleinement leur potentiel. Elles les affaiblissent et leur nuisent dans la possibilité même de vivre en société. Faute d'accorder l'attention nécessaire à ces conditions sociales préalables, il n'y a aucune raison de croire que des services sociaux uniquement réactifs aux problèmes selon une orientation thérapeutique auront un impact sur les problèmes fondamentaux.

Trois conditions particulières de vulnérabilité sont mises en évidence dans le rapport :

- les situations de handicap, c'est-à-dire les déficiences ou les incapacités qui placent la personne dans une situation de désavantage social ayant pour effet de la limiter ou de l'empêcher d'exercer certains rôles sociaux;

- la pauvreté, dont le lien avec les autres problèmes sociaux a déjà été souligné : c'est une condition de vie résultant de l'absence ou de l'insuffisance de ressources matérielles et financières;

- l'absence ou l'insuffisance des réseaux d'intégration sociale tels qu'ils se manifestent dans l'isolement, la solitude, l'impossibilité de recourir à un niveau social naturel ou à un réseau externe $d^{\prime}$ 'intégration sociale ${ }^{26}$.

Les situations de handicap, la pauvreté et l'insuffisance des réseaux d'intégration ont un potentiel élevé d'association avec la présence des problèmes fondamentaux. C'est pourquoi il est nécessaire de dépasser la seule conception des problèmes sociaux centrée sur les manifestations de situations graves, intolérables, inacceptables et urgentes pour les situer en interface avec certaines caractéristiques propres à certaines populations particulières. On n'est plus uniquement en présence des incapacités des individus à faire face à la vie en société, mais on est aux prises avec des insuffisances systémiques 
de la société concernant des situations dans lesquelles se trouvent des parties importantes de la population du Québec.

Malheureusement, une telle conception des réalités à l'origine des problèmes sociaux est beaucoup moins mobilisatrice de ressources, et ses possibilités d'insertion dans les politiques sociales, beaucoup moins faciles. En fait, définir les problèmes sociaux en y incorporant des conditions de vulnérabilité dans lesquelles vivent les enfants, la jeunesse, les familles monoparentales et les personnes âgées, est beaucoup plus difficile à faire accepter par l'opinion publique. Dans notre société, on obtient plus facilement des fonds en parlant des situations extrêmes ou d'urgence, que lorsqu'on fait ressortir la situation de segments importants de la population qui pourtant se trouvent à un degré très élevé en situation potentielle de problèmes. D'ailleurs, comme l'a montré récemment Michael Lipsky ${ }^{27}$, différents leaders des organismes tant communautaires que publics ont souvent tendance à se contenter d'approches faisant ressortir l'urgence des situations. Elles sont peut-être plus efficaces à court terme pour l'obtention de ressources, mais elles ont la faiblesse d'être fragmentées et sans perspective d'ensemble.

Même si la Commission n'a pas incorporé les conditions de vulnérabilité dans ses recommandations, leur inclusion dans son analyse des problèmes sociaux ouvre la voie à une définition des orientations de la politique en matière de services sociaux qui dépasse la seule réponse aux problèmes vus comme des urgences ou des menaces immédiates pour la société. Dans cette perspective, les services sociaux peuvent être définis pour répondre aux besoins de segments importants de la population québécoise en amont de l'apparition des situations graves que constituent les problèmes sociaux fondamentaux ${ }^{28}$. Ce faisant, il y a possibilité de définir une politique réelle de la famille qui se fonde d'abord sur les besoins des enfants, en tenant compte en particulier de la surcharge des familles monoparentales et des familles qui comptent une personne handicapée. De la même manière, l'intégration des jeunes dans les différentes sphères de la vie en société devrait aussi être l'objet de l'attention des pouvoirs publics. Quant au maintien maximal de toutes les possibilités d'insertion sociale des aînés, elle saute aux yeux à la seule considération de l'augmentation du nombre et de l'importance des personnes âgées dans la société québécoise.

\section{Les difficultés courantes de fonctionnement en société}

Le dernier degré de problèmes défini par la Commission est celui des difficultés courantes de fonctionnement en société29. En fait, 
l'insertion de ces difficultés courantes constitue une redéfinition même de la problématique de base concernant les réponses à fournir aux problèmes sociaux. Elle se fonde sur la reconnaissance du fait que dans la société québécoise, la famille, le voisinage ou la paroisse, comme structure traditionnelle immédiate d'assistance aux personnes, sont dans bien des cas devenus caducs pour aider ces dernières à faire face à leurs difficultés. Même si de nouvelles formes d'entraide et de services sont apparues et sont devenues des sources importantes d'appui à la solution des difficultés des personnes, il n'en demeure pas moins que, dans certaines circonstances, elles sont insuffisantes et quelquefois inappropriées : elles peuvent même contribuer à accentuer les problèmes qu'elles veulent solutionner.

Tout en faisant ressortir une série de besoins qui découlent de certaines circonstances de la vie familiale et conjugale et en mettant en évidence certains besoins sociaux liés aux problèmes de santé, d'éducation et de travail, la Commission insiste pour que les problèmes courants aient leur solution dans leur milieu d'origine. Elle fait ainsi ressortir quatre besoins principaux auxquels les services sociaux doivent répondre :

- le besoin de disposer d'un endroit où la personne peut s'adresser pour exposer son problème en toute quiétude et où la confidentialité est garantie;

- le besoin de connaître les ressources existantes susceptibles d'aider à faire face aux difficultés;

- le besoin de connaître la nature, l'étendue et les répercussions possibles de la situation;

- le besoin de connaître les possibilités de solution et les moyens à prendre en regard des problèmes ${ }^{30}$.

On reconnaît donc l'existence de besoins dans la vie de tous les jours. Des difficultés surgissent continuellement dans les situations vécues par les personnes. II s'agit de difficultés de toutes sortes qui affectent la capacité de fonctionner de la personne, ses relations avec les autres, son insertion dans son milieu immédiat ou son intégration sociale.

Des lieux précis doivent être mis en place pour fournir l'écoute à ces personnes, leur assurer qu'elles pourront y parler en toute tranquillité des difficultés vécues et qu'elles auront le respect et la confidentialité voulus lorsqu'elles ne sont pas en mesure de le faire avec leurs proches ou lorsque ces derniers ne peuvent leur apporter I'aide souhaitée. De la même manière, face aux dédales et aux complications de l'organisation du système des ressources, les personnes ne sont pas souvent en mesure de s'orienter vers la voie la plus directe qui leur permettra de trouver les solutions requises et efficaces à leurs difficultés courantes. En conséquence, au ras de sol 
de la réalité sociale quotidienne, il y a cette problématique de base qui consiste à rendre l'organisation sociale sensitive et capable d'apporter une réponse aux difficultés vécues en matière de socialité.

\section{Conclusion}

La conception des problèmes sociaux qu'on retrouve dans le rapport Rochon part d'une vision macroscopique de la société québécoise. Toutefois, malgré cela, compte tenu du mandat circonscrit de la Commission, certains problèmes sociaux, notamment ceux de la pauvreté et de l'insécurité économique de plusieurs groupes de la population québécoise, ont été en bonne partie mis de côté dans l'analyse des problèmes sociaux.

L'analyse des problèmes sociaux faite par la Commission a une portée qui va au-delà de la simple identification des pathologies. En matière de santé, en plus des maladies, on met l'insistance sur les racines biologiques des problèmes, leurs liens avec les habitudes de vie ou les comportements et leur dimension environnementale. En conséquence, les politiques à définir en matière de santé se situent dans une perspective beaucoup plus globale que la seule perspective de la lutte aux maladies ou à leur prévention pour s'ouvrir au social et à l'environnement.

De la même manière, il y a éclatement de la conception des problèmes auxquels s'adressent les services sociaux. Ce ne sont plus uniquement les problèmes de violence contre les personnes (abus, négligence, agressions, mauvais traitements ou problèmes de déviance et de mésadaptation sociale) qui sont au cœur des services sociaux. On leur ajoute certains problèmes clés d'intégration sociale : les carences du lien parents-enfants, la surcharge de certaines familles monoparentales, les difficultés sociales liées à l'autonomie fonctionnelle et les problèmes d'intégration à la société québécoise que vivent les immigrants. La Commission va encore plus loin pour définir des problèmes à partir des conditions de vulnérabilité que sont les situations de handicap, la pauvreté et l'inexistence ou l'insuffisance des réseaux d'intégration. Elle débouche sur la nécessité pour les services sociaux d'être des ressources pour aider les personnes à faire face à leurs difficultés courantes de fonctionnement en société. En conséquence, les politiques en matière de services sociaux qui en découleront pourraient devenir beaucoup plus proactives et axées sur les besoins de la population plutôt que réactives aux seules situations détériorées et définies par leur caractère d'urgence. Pourtant, la Commission ne s'est pas rendue jusque-là dans ses recommandations en matière de problèmes sociaux. Ces dernières concernent uni- 
quement les trois séries de problèmes sociaux fondamentaux. Bien plus, on peut se demander jusqu'à quel point les orientations ministérielles proposées ne sont pas encore plus restreintes dans leur conception des problèmes sociaux qui doivent être l'objet d'attention.

Force est de conclure, enfin, que si la définition des problèmes en matière de santé s'inspire d'une forte tradition de recherche, il $n^{\prime}$ en est pas de même du côté des services sociaux. C'est un besoin urgent d'y accentuer les travaux de recherche pour éclairer davantage les choix à faire et connaître les effets des actions entreprises.

\section{Notes et références}

* L'auteur a été conseiller scientifique à la Commission Rochon.

${ }^{1}$ Du nom du président de la Commission d'enquête sur les services de santé et les services sociaux : Jean Rochon.

${ }^{2}$ Rapport de la Commission d'enquête sur les services de santé et les services sociaux. Québec: Les Publications du Québec, 1988. (Ci-après appelé Rapport Rochon).

${ }^{3}$ Plusieurs auteurs ont traité cette question au cours des dernières années. On retrouvera dans le volume de D.L. Badden (éd.), The Social Contract Revisited, Washington, D.C. : Urban Institute, 1984, une synthèse des principaux éléments de la remise en question.

${ }^{4}$ Les volumes américains récents traitant des problèmes sociaux font la plupart du temps la distinction entre les problèmes globaux ou mondiaux (les mégaproblèmes), les problèmes structurels ou sociétaux (les macro-problèmes) et les problèmes individuels ou macroscopiques. Voir par exemple : Brian J. Jones, Bernard J. Galagher III et Joseph A. McFalls Jr., Social Problems - Issues, Opinions and Solutions. New York, McGraw-Hill (1988); D. Stanley Eitzen et Maxime Baca Zinn, Social Problems ( $4^{\mathrm{e}}$ édition). Boston : Allyn and Bacon (1989).

${ }^{5}$ Le premier chapitre du Rapport Rochon est consacré à cette analyse. Voir Rapport Rochon, op. cit. : 3-38.

${ }^{6}$ Rapport Rochon, op. cit. : 34.

'Etzioni, A. (1976). Social Problems. Englewood Cliffs, N.J. : Prentice Hall, p. 34 (traduction de l'auteur).

${ }^{8}$ Le décret 57-86 du 29 janvier 1986 définissait le mandat de la Commission comme suit :

a) Évaluer le fonctionnement et le financement du système des services de santé et des services sociaux en regard de ses objectifs et plus particulièrement :

1. Les fonctions des différentes parties du système des services de santé et des services sociaux incluant :

- les responsabilités réciproques du ministère de la Santé et des Services sociaux, des conseils régionaux et des établissements;

- la coordination des niveaux de décision;

- le rôle des professionnels au sein du système; 
- les mécanismes de participation du milieu et de concertation avec les principaux collaborateurs externes au système de santé et de services sociaux.

2. Le financement des services de santé et des services sociaux en tenant compte notamment :

- des facteurs influençant l'offre et la demande de services;

- du développement technologique;

- du niveau et des modes possibles de financement;

- du processus de décision pour l'allocation des ressources;

- des mécanismes d'évaluation et de contrôle.

b) Étudier les diverses solutions possibles aux différents problèmes que connaît le système des services de santé et des services sociaux.

c) Faire au Gouvernement les recommandations qui lui semblent les plus appropriées pour assurer le maintien et le développement des services de santé et des services sociaux.

${ }^{9}$ Dans son rapport, la Commission Castonguay-Nepveu avait consacré un volume complet à la question de la sécurité du revenu et fait des recommandations précises à ce sujet. Voir Rapport de la Commission d'enquête sur la santé et le bien-être social - Volume $V$ - La sécurité du revenu. Québec : Gouvernement du Québec, 1971.

${ }^{10}$ Rapport Rochon, op. cit. : 85.

${ }^{11}$ Rapport Rochon, op. cit. : 126.

${ }^{12}$ Rapport Rochon, op, cit. : 41-92.

${ }^{13}$ Rapport Rochon, op. cit. : 43. Nous avons exclu de notre analyse le volet de l'organisation du système de soins, car il s'agit d'abord d'un problème de gestion de la santé.

${ }^{14}$ Rapport Rochon, op. cit. : 75.

${ }^{15}$ Voir le chapitre consacré aux problèmes de santé par D. Stanley Eitzen et Maxime Baca Zinn, op. cit. : 93-118.

${ }^{16}$ Rapport Rochon, op. cit. : 95.

(17)André Beaudoin. Services sociaux à l'enfance, à la famille, aux personnes âgées et dans le domaine de la santé. L'état de la situation au Québec. Université Laval, École de service social, Laboratoire de recherche, 1988 : 1120. On trouvera une élaboration plus détaillée de cette notion à cet endroit.

${ }^{18}$ Rapport Rochon, op. cit. : 93.

${ }^{19}$ Rapport Rochon, op. cit. : 109.

${ }^{20}$ Ibidem.

${ }^{21}$ Rapport Rochon, op. cit. : 115-124.

${ }^{22}$ Brunet, Jacques et al., Rapport du Comité de réflexion et d'analyse des services dispensés par les CLSC (Rapport Brunet). Québec: Ministère de la santé et des services sociaux, 1987.

${ }^{23}$ Voir l'analyse faite sur cette question par Marie Drolet : " La réaliser sans la nommer ? La réorientation des CLSC à travers la notion de risque ". Revue canadienne de politique sociale, $\mathrm{n}^{\circ} 24,1989$ : 45-58.

${ }^{24}$ Rapport Rochon, op. cit. : 125.

${ }^{25}$ Voir I'analyse faite par Alfred J. Kahn à ce sujet : "Social Problems and Issues: Theories and Definition ». Encyclopediae of Social Work. Ann Minaham (éd.), 18 édition, Silver Spring, Maryland: NASW, 1987, vol. 2 : 639-640. 
${ }^{26}$ Rapport Rochon, op. cit. : 125-127.

${ }^{27}$ Cette dimension est explorée par Michael Lipsky, Street-Level Bureaucracy. New York : Russel Sage, 1987 : 136-139.

${ }^{28}$ L'analyse faite à cet égard par Lipsky et Rathgeb Smith est particulièrement révélatrice. Michael Lipsky et Steven Rathgeb Smith, "When Social Problems are Defined as Emergencies ». Social Service Review, vol. 63, n 1, 1989 : 5-15.

${ }^{29}$ Rapport Rochon, op. cit. : 133-137.

${ }^{30}$ Rapport Rochon, op. cit.': 134. 